\title{
Exact analysis of entanglement in gapped quantum spin chains
}

\author{
Hosho Katsura, ${ }^{1, *}$ Takaaki Hirano, ${ }^{1, \dagger}$ and Yasuhiro Hatsugai ${ }^{1,2, \$}$ \\ ${ }^{1}$ Department of Applied Physics, The University of Tokyo, 7-3-1, Hongo, Bunkyo-ku, Tokyo 113-8656, Japan \\ ${ }^{2}$ Institute of Physics, University of Tsukuba, 1-1-1, Tennodai, Tsukua, Ibaraki, 305-8571, Japan
}

(Received 13 April 2007; published 3 July 2007)

\begin{abstract}
We investigate the entanglement properties of the valence-bond-solid states with generic integer spin $S$. Using the Schwinger boson representation of the valence-bond-solid states, the entanglement entropy, the von Neumann entropy of a subsystem, is obtained exactly and its relationship with the usual correlation function is clarified. The saturation value of the entanglement entropy, $2 \log _{2}(S+1)$, is derived explicitly and is interpreted in terms of the edge-state picture. The validity of our analytical results and the edge-state picture is numerically confirmed. We also propose an application of the edge state as a qubit for quantum computation.
\end{abstract}

DOI: 10.1103/PhysRevB.76.012401

PACS number(s): 75.10.Pq, 03.65.Ud, 03.67.Mn, 05.70.Jk

Entanglement properties of quantum spin systems have been attracting much attention in quantum information theory and condensed-matter physics. The entanglement entropy (EE), the von Neumann entropy of the reduced density matrix of a subsystem, is a measure to quantify how much entangled a many-body ground state is. Recently, the EE has been used to investigate the nature of quantum ground states such as the quantum phase transition and topological and/or quantum order. ${ }^{1-5}$ Vidal et al. ${ }^{1}$ conjectured that the EE of a large block of spins in gapped spin chains reaches saturation while that in critical spin chains shows a logarithmic divergence.

In this Brief Report, we study the EE of gapped quantum spin chains with arbitrary integer spin. After the Haldane conjecture that integer-spin antiferromagnetic Heisenberg chains have a finite gap, ${ }^{6,7}$ Affleck, Kennedy, Lieb, and Tasaki (AKLT) proposed the valence-bond-solid (VBS) state which enables us to understand ground-state properties of the Haldane gap systems. ${ }^{8,9}$ The VBS is now attracting renewed interest from the viewpoint of quantum information theory. For example, universal quantum computation based on the VBS states has been proposed. ${ }^{10}$

While the entanglement properties in $S=1 \mathrm{VBS}$ has been extensively studied in Refs. 12 and 13, we investigate the EE in generic VBS states with arbitrary integer spin $S$. We stress that there exist not only $S=1$ antiferromagnetic Heisenberg chains ${ }^{14,15}$ but also an $S=2$ chain $\left[\mathrm{MnCl}_{3}\right.$ (bipy)], ${ }^{16}$ in which the presence of the Haldane gap has been experimentally confirmed. We give the exact form of the EE in generic VBS states in this Brief Report. Then, we explicitly confirm that the part of the conjecture proposed by Vidal et al. is true for all integer-spin VBS chains. The relationship between the EE and the correlation function is clarified and the physical meaning of the EE in gapped models is established. We also make a comparison between the analytical results for VBS chains and the numerical results for higher-spin antiferromagnetic Heisenberg chains. The obtained results indicate that the edge-state picture is valid not only for $S=1$ Haldane chains but also for all the other integer-spin $S$ chains. This is a typical consequence of the nontrivial topological and/or quantum orders, where characteristic features are hidden in the bulk and appear only near the boundaries and impurities. ${ }^{17}$ We also discuss a potential application of the edge states as qubits for quantum computation.
Let us start with the Schwinger boson representation of generic VBS states. The spin operators are represented by the Schwinger bosons as $S_{j}^{+}=a_{j}^{\dagger} b_{j}, S_{j}^{-}=b_{j}^{\dagger} a_{j}$, and $S_{j}^{z}=\left(a_{j}^{\dagger} a_{j}\right.$ $\left.-b_{j}^{\dagger} b_{j}\right) / 2$, where $a_{j}^{\dagger}$ and $b_{j}^{\dagger}$ satisfy $\left[a_{i}, a_{j}^{\dagger}\right]=\left[b_{i}, b_{j}^{\dagger}\right]=\delta_{i j}$ with the all the other commutators vanishing. ${ }^{18}$ To reproduce the dimension of the spin $S$ Hilbert space at each site, we must impose the constraint that the total boson occupation number $a_{j}^{\dagger} a_{j}+b_{j}^{\dagger} b_{j}=2 S$. Using the Schwinger boson representation, the spin $S$ VBS state with two spin $S / 2$ 's on the boundary is written as

$$
|\mathrm{VBS}\rangle=\prod_{j=0}^{N}\left(a_{j}^{\dagger} b_{j+1}^{\dagger}-b_{j}^{\dagger} a_{j+1}^{\dagger}\right)^{S}|\mathrm{vac}\rangle,
$$

where $j=1,2, \ldots, N$ are bulk sites and 0 and $N+1$ are end sites. $B_{i j} \equiv a_{i}^{\dagger} b_{j}^{\dagger}-b_{i}^{\dagger} a_{j}^{\dagger}$ is a creation operator for the valence bond between $i$ and $j .{ }^{19}$ The VBS state [Eq. (1)] is a zeroenergy ground state of the following Hamiltonian:

$$
H=\sum_{j=1}^{N-1} \sum_{J=S+1}^{2 S} A_{J} P_{j, j+1}^{J}+\pi_{0,1}+\pi_{N, N+1},
$$

where the projection operator $P_{j, j+1}^{J}$ projects the bond spin $\vec{J}_{j, j+1}=\vec{S}_{j}+\vec{S}_{j+1}$ onto the subspace of magnitude $J$. Here the coefficient $A_{J}$ can be an arbitrary positive value. The boundary terms describing the interaction between spin $S / 2$ and spin $S$ are explicitly written as

$$
\pi_{0,1}=\sum_{J=S / 2+1}^{3 S / 2} B_{J} P_{0,1}^{J}, \quad \pi_{N, N+1}=\sum_{J=S / 2+1}^{3 S / 2} B_{J} P_{N, N+1}^{J},
$$

with $B_{J}>0$. In order to calculate reduced density matrices, it is convenient to introduce a spin coherent state. For a point $\hat{\Omega}=(\sin \theta \cos \phi, \sin \theta \sin \phi, \cos \theta)$ on the unit sphere, the spin coherent state at each site is defined as

$$
|\hat{\Omega}\rangle=\frac{\left(u a^{\dagger}+v b^{\dagger}\right)^{2 S}}{\sqrt{(2 S) !}}|0\rangle,
$$

where $(u, v)=\left(\cos (\theta / 2) e^{i \phi / 2}, \sin (\theta / 2) e^{-i \phi / 2}\right)$ are spinor coordinates. Here, we have already fixed the $U(1)$ gauge degree of freedom since it has no physical content. Using $|\hat{\Omega}\rangle$, the 
trace of any operator $\mathcal{O}$ is written as $\operatorname{Tr} \mathcal{O}$ $=\frac{2 S+1}{4 \pi} \int d \hat{\Omega}\langle\hat{\Omega}|\mathcal{O}| \hat{\Omega}\rangle$.

Let us now calculate the EE of a block of $L$ contiguous bulk spins in the VBS state [Eq. (1)]. For the density matrix of our ground state $\rho=|\mathrm{VBS}\rangle\langle\mathrm{VBS}| /\langle\mathrm{VBS} \mid \mathrm{VBS}\rangle$, the reduced density matrix of the block of $L$ contiguous bulk spins is defined as $\rho_{L}=\operatorname{Tr}_{\mathcal{B}_{L}}^{-} \rho$. Here $\mathcal{B}_{L}$ is a block of $L$ spins and
$\overline{\mathcal{B}}_{L}$ is its complement. The EE $\mathcal{S}_{L}=-\operatorname{Tr}_{\mathcal{B}_{L}} \rho_{L} \log _{2} \rho_{L}$ is determined by eigenvalues of $\rho_{L}$. Suppose that the block of $L$ contiguous spins starting from site $k$ and stretching up to $k$ $+L-1$, where $k \geqslant 1$ and $k+L-1 \leqslant N$. To obtain the reduced density matrix $\rho_{L}$, we take the trace over the sites $j$ $=0,1, \ldots, k-1$ and $j=k+L, \ldots, N, N+1$. Using the spin coherent-state representation, $\rho_{L}$ is formally written as

$$
\rho_{L}=\frac{\int\left(\prod_{j \in \overline{\mathcal{B}}_{L}} \frac{d \hat{\Omega}_{j}}{4 \pi}\right) \prod_{j=0}^{k-1}\left(\frac{1-\hat{\Omega}_{j} \cdot \hat{\Omega}_{j+1}}{2}\right)^{S} \prod_{l=k+L}^{N}\left(\frac{1-\hat{\Omega}_{l} \cdot \hat{\Omega}_{l+1}}{2}\right)^{S} Q_{k}^{\dagger} P_{k+L-1}^{\dagger}\left|\mathrm{VBS}_{L}\right\rangle\left\langle\operatorname{VBS}_{L}\right| Q_{k} P_{k+L-1}}{(2 S+1)^{L}[(2 S) !]^{L} \int\left(\prod_{j \in \mathcal{B}_{L} \overline{\mathcal{B}}_{L}} \frac{d \hat{\Omega}_{j}}{4 \pi}\right) \prod_{j=0}^{N}\left(\frac{1-\hat{\Omega}_{j} \cdot \hat{\Omega}_{j+1}}{2}\right)^{S}},
$$

where boundary operators and a block of VBS state with length $L$ are defined as $Q_{k}=\left(u_{k-1} b_{k}-v_{k-1} a_{k}\right)^{S}, \quad P_{k+L-1}$ $=\left(a_{k+L-1} v_{k+L}-b_{k+L-1} u_{k+L}\right)^{S}, \quad$ and $\quad\left|\operatorname{VBS}_{L}\right\rangle=\prod_{j=k}^{k+L-2}\left(a_{j}^{\dagger} b_{j+1}^{\dagger}\right.$ $\left.-b_{j}^{\dagger} a_{j+1}^{\dagger}\right)^{S}\left|\operatorname{vac}_{L}\right\rangle$, respectively. Here we have already used the following relation: $\left\langle 0\left|a^{S-l} b^{S+l}\right| \hat{\Omega}\right\rangle=\sqrt{(2 S) !} u^{S-l} v^{S+l}$. In Eq. (5), the integrals over $\hat{\Omega}_{k-l-1}(l=1,2, \ldots, k-1)$ can be performed by regarding $\hat{\Omega}_{k-l}$ as a polar axis. The same holds for $\hat{\Omega}_{k+L+m}$ $(m=1,2, \ldots, N-L-k+1)$. After integrating over these variables, we immediately notice that the reduced density matrix $\rho_{L}$ does not depend on both the starting site $k$ and the total length $N$. The same property for $S=1 \mathrm{VBS}$ has been proved in Ref. 13 by another approach, i.e., using the special property of maximally entangled states. The coherent-state approach, however, allows us to generalize this result for more complicated cases. For example, we can also prove that the EE does not depend on the whole size of a VBS state on a two-dimensional Cayley tree ${ }^{20}$ by using the coherent-state representation.

Since the reduced density matrix does not depend on both $k$ and $N$, we can set $N=L$ without loss of generality. The following remarkable property makes it easier to calculate the EE of $L$ contiguous spins: $\mathcal{S}_{L}=\mathcal{S}_{\hat{L}} \equiv-\operatorname{Tr}_{\overline{\mathcal{B}}_{L}} \rho_{\hat{L}} \log _{2} \rho_{\hat{L}}$, where $\rho_{L} \equiv \operatorname{Tr}_{\mathcal{B}_{L}} \rho$. One can easily show this by using the Schmidt decomposition. Then, all we have to do is to obtain the eigenvalues of the reduced density matrix of two end spin S/2's,

$\rho_{\hat{L}}$

$$
=\frac{\int \prod_{j=1}^{L} \frac{d \hat{\Omega}_{j}}{4 \pi} \prod_{k=1}^{L-1}\left(\frac{1-\hat{\Omega}_{k} \cdot \hat{\Omega}_{k+1}}{2}\right)^{S} P_{0}^{\dagger} Q_{L+1}^{\dagger}|\operatorname{vac}\rangle\langle\operatorname{vac}| P_{0} Q_{L+1}}{(S !)^{2} \int\left(\prod_{j=1}^{L} \frac{d \hat{\Omega}_{j}}{4 \pi}\right) \prod_{k=1}^{L-1}\left(\frac{1-\hat{\Omega}_{k} \cdot \hat{\Omega}_{k+1}}{2}\right)^{S}},
$$

where $|\operatorname{vac}\rangle\langle\operatorname{vac}|\equiv| 0\rangle_{0}\langle 0|\otimes| 0\rangle_{L+1}\langle 0|$. The state $P_{0}^{\dagger}|0\rangle_{0}$ in the numerator of Eq. (3) is explicitly given by $\left(a_{0}^{\dagger} v_{1}^{*}-b_{0}^{\dagger} u_{1}^{*}\right)^{S}|0\rangle$. From the definition of the spinor coordinates, we notice that $(u, v)$ changes to $\left(i v^{*},-i u^{*}\right)$ when we change variables from $(\theta, \phi)$ to $(\pi-\theta, \phi+\pi)$. Then, we can rewrite $P_{0}^{\dagger}|0\rangle_{0}$ as ( $-i)^{S} \sqrt{S !}\left|-\hat{\Omega}_{1}\right\rangle_{0}$. In the same way, $Q_{L+1}^{\dagger}|0\rangle_{L+1}$ can be rewritten as $i \sqrt{S !}\left|-\hat{\Omega}_{L}\right\rangle_{L+1}$. Substituting these results into Eq. (3) and changing the variables of integration from $\hat{\Omega}_{j}$ to $-\hat{\Omega}_{j}(j$ $=1,2, \ldots, L)$, we obtain

$$
\begin{aligned}
& \rho_{\hat{L}} \\
& =\frac{\int \prod_{j=1}^{L} \frac{d \hat{\Omega}_{j}}{4 \pi} \prod_{k=1}^{L-1}\left(\frac{1-\hat{\Omega}_{k} \cdot \hat{\Omega}_{k+1}}{2}\right)^{S}\left|\hat{\Omega}_{1}\right\rangle_{0}\left\langle\hat{\Omega}_{1}|\otimes| \hat{\Omega}_{L}\right\rangle_{L+1}\left\langle\hat{\Omega}_{L}\right|}{\int\left(\prod_{j=1}^{L} \frac{d \hat{\Omega}_{j}}{4 \pi}\right)_{k=1}^{L-1}\left(\frac{1-\hat{\Omega}_{k} \cdot \hat{\Omega}_{k+1}}{2}\right)^{S}} .
\end{aligned}
$$

Now the physical meaning of $\rho_{\hat{L}}$ is quite clear. Equation (4) can be regarded as a correlation function between density matrices $\left|\hat{\Omega}_{1}\right\rangle_{0}\left\langle\hat{\Omega}_{1}\right|$ and $\left|\hat{\Omega}_{L}\right\rangle_{L+1}\left\langle\hat{\Omega}_{L}\right|$. More precisely, the matrix elements of $\rho_{\hat{L}}$ are completely determined by the twopoint correlation functions of the corresponding onedimensional classical statistical model. ${ }^{19}$ This can be checked by using the binomial expansion of $P_{0}$ and $Q_{L+1}$. While this interpretation enables us to understand the relation between the EE and the correlation functions, it is more convenient to use form (4) for the calculation of the EE.

From now on, we follow Ref. 11 and obtain the eigenvalues of $\rho_{\hat{L}}$. In Eq. (4), $T_{k, k+1}=\left(\frac{1-\hat{\Omega}_{k} \cdot \hat{\Omega}_{k+1}}{2}\right)^{S}$ acts as a transfer matrix of the corresponding classical statistical model. Expanding $T_{k, k+1}$ in terms of Legendre polynomials and using the addition theorem for spherical harmonics, the transfer matrix can be rewritten as 


$$
T_{k, k+1}=\frac{4 \pi}{S+1} \sum_{l=0}^{S} \lambda(l) \sum_{m=-l}^{l} Y_{l}^{m}\left(\hat{\Omega}_{k}\right) \overline{Y_{l}^{m}\left(\hat{\Omega}_{k+1}\right)}
$$

with $\lambda(l) \equiv(-1)^{l} S !(S+1) ! /[(S-l) !(S+l+1) !]$. Then, we substitute Eq. (5) into Eq. (4), recall the orthonormality of spherical harmonics, i.e., $\int d \hat{\Omega} \overline{Y_{l}^{m}(\hat{\Omega})} Y_{l^{\prime}}^{m^{\prime}}(\hat{\Omega})=\delta_{l l^{\prime}} \delta^{n m^{\prime}}$, and obtain

$$
\rho_{\hat{L}}=\frac{4 \pi}{(S+1)^{2}} \sum_{l=0}^{S} \lambda(l)^{L-1} \sum_{m=-l}^{l}\left[T_{l}^{(m)} \otimes\left(T_{l}^{(m)}\right)^{\dagger}\right],
$$

where irreducible $l$ th order spherical tensor operators $T_{l}^{(m)}(m=-l,-l+1, \ldots, l)$ are defined as $T_{l}^{(m)}$ $\equiv \frac{2 \cdot S / 2+1}{4 \pi} \int d \hat{\Omega}|\hat{\Omega}\rangle Y_{l}^{m}(\hat{\Omega})\langle\hat{\Omega}|$. We should note here that $T_{l}^{(m)}$ acts on the Hilbert space of the left-end spin $S / 2$, while $\left(T_{l}^{(m)}\right)^{\dagger}$ acts on that of the right-end spin $S / 2$. Let us now introduce the following formula found in Ref. 11:

$$
\sum_{m=-l}^{l}\left[T_{l}^{(m)} \otimes\left(T_{l}^{(m)}\right)^{\dagger}\right]=I_{l}\left(\vec{S}_{0} \cdot \vec{S}_{L+1}\right),
$$

where $\vec{S}_{0}$ and $\vec{S}_{L+1}$ denote the left-end and right-end spin $S / 2$ 's, respectively. Here $I_{j}(X)$ is a $j$ th order polynomial in $X$ and determined by the following recursion relation:

$$
\begin{aligned}
I_{j+1}(X)= & \frac{2 j+3}{(S+j+2)^{2}}\left(\frac{4 X}{j+1}+j\right) I_{j}(X) \\
& -\frac{j}{j+1} \frac{2 j+3}{2 j-1}\left(\frac{S-j+1}{S+j+2}\right)^{2} I_{j-1}(X),
\end{aligned}
$$

with $I_{0}(X)=\frac{1}{4 \pi}, I_{1}(X)=\frac{3}{4 \pi} \frac{X}{(S / 2+1)^{2}}$. The isotropic two site tensor operators $I_{j}\left(\vec{S}_{0} \cdot \vec{S}_{L+1}\right)(j=0,1, \ldots, S)$ are mutually orthogonal with respect to the trace inner product $\operatorname{Tr}_{0, L+1}\left(I_{j} I_{k}\right)$. Since Eq. (6) is completely determined by the polynomials in $\vec{S}_{0} \cdot \vec{S}_{L+1}$, the reduced density matrix $\rho_{\hat{L}}$ is diagonal in the basis which diagonalizes the total spin operator $\vec{J}_{0, L+1}=\vec{S}_{0}$ $+\vec{S}_{L+1}$. Therefore, the eigenvalues of $\rho_{\hat{L}}$ are given by

$$
\rho_{\hat{L}}(J)=\frac{4 \pi}{(S+1)^{2}} \sum_{l=0}^{S} \lambda(l)^{L-1} I_{l}\left(\frac{1}{2} J(J+1)-\frac{S}{2}\left(\frac{S}{2}+1\right)\right),
$$

where $J(=0,1,2, \ldots, S)$ is a magnitude of the total spin and each $\rho_{\hat{L}}(J)$ is $(2 J+1)$-fold degenerate. Finally, the EE of a block of $L$ contiguous bulk spins is explicitly written as

$$
\mathcal{S}_{L}=-\sum_{J=0}^{S}(2 J+1) \rho_{\hat{L}}(J) \log _{2} \rho_{\hat{L}}(J) .
$$

Since the reduced density matrix $\rho_{\hat{L}}$ approaches an $(S+1)^{2}$-dimensional identity matrix in the thermodynamic limit $L \rightarrow \infty$, we can see that $\mathcal{S}_{L} \leqslant 2 \log _{2}(S+1) \equiv \mathcal{S}_{\infty}(S)$ and approaches this upper bound exponentially fast in $L$. This saturation can be observed in Fig. 1, where the EE $\mathcal{S}_{L}$ for various spin- $S$ VBS chains are plotted as a function of the block size $L$. Here, we confirm that the conjecture proposed by Vidal et al. is valid for all integer-spin VBS chains.

Next we make a comparison between the above results for

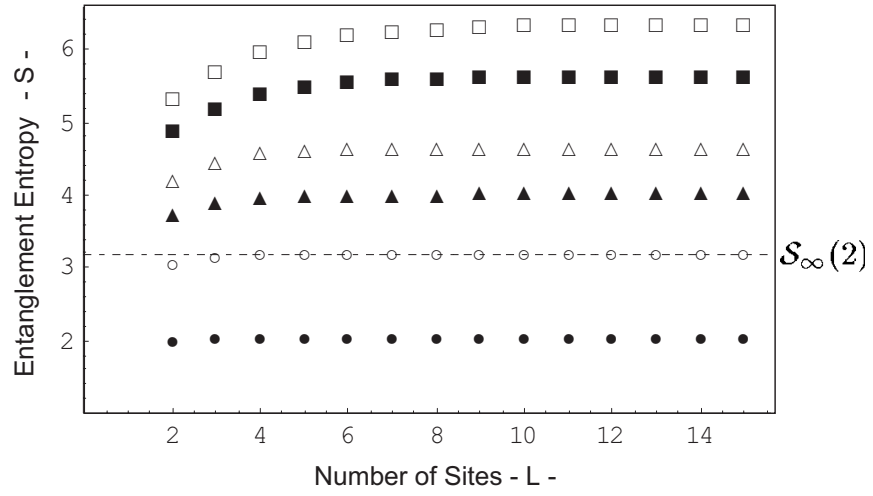

FIG. 1. The EE for $S=1(\bullet), S=2(\bigcirc), S=3(\mathbf{\Delta}), S=4(\triangle), S$ $=6(\boldsymbol{\square})$, and $S=8(\square)$ VBS chains as a function of the block size $L$. The broken line indicates the saturation value $\mathcal{S}_{\infty}(2)$.

the VBS chains and numerical results for the integer-spin Heisenberg models. Since $S=1$ systems have recently been extensively studied, ${ }^{24}$ we study numerically the EE and the energy spectra of the $S=2$ Heisenberg model and its continuous deformations. One of the simplest $S=2$ Hamiltonian which interpolates between these two models can be written as

$$
H=\sum_{i=1}^{N} \vec{S}_{i} \cdot \vec{S}_{i+1}+\alpha\left[\frac{2}{9}\left(\vec{S}_{i} \cdot \vec{S}_{i+1}\right)^{2}+\frac{1}{63}\left(\vec{S}_{i} \cdot \vec{S}_{i+1}\right)^{3}+\frac{10}{7}\right],
$$

where $\alpha=0$ and $\alpha=1$ correspond to the Heisenberg model and the $S=2$ AKLT model, respectively.

The edge-state picture ${ }^{21}$ in general $S$ Haldane systems allows us to interpret the spectra as follows. The low-lying $(S+1)$ multiplets have $\left(2 S_{\text {total }}+1\right)$-fold degeneracy in each sector when the system has open boundaries. These generalized Kennedy triplet states are almost degenerate and are completely degenerate at the AKLT point. This can be understood from the VBS picture. It would be valid for the Heisenberg model by some results from numerical calculations. 22,23 Let us now show that the ground-state properties remain unchanged through the adiabatic continuation from the AKLT to the Heisenberg model. Figure 2(a) shows the $\alpha$ dependence of the energy gaps between the ground and the lowest two excited states computed by exact diagonalizations of the system of $N=10$ sites with periodic boundary conditions. There is no level crossing between the ground state and the first excited state, which suggests that the low-energy behaviors of the system are adiabatically equivalent with each other in this parameter region.

Finally, let us discuss the EE in our system. The obtained results of the EE from exact diagonalizations are shown in Fig. 2(b). The EE at the AKLT point has a tendency to converge to the value $\mathcal{S}_{\infty}(2)=2 \log _{2} 3=3.16993$ as the system size increases. This value coincides with our analytically calculated one with open boundary conditions (see Fig. 1). The lower bound of the $\mathrm{EE}$ in the calculated region is given by $\mathcal{S} \geqslant 2 \log _{2} 3$, and this is the contribution from the boundaries of the system created by taking partial trace over the subsystem. This lower bound is equal to the EE at the AKLT point. Taking the edge-state picture into account, we can see 


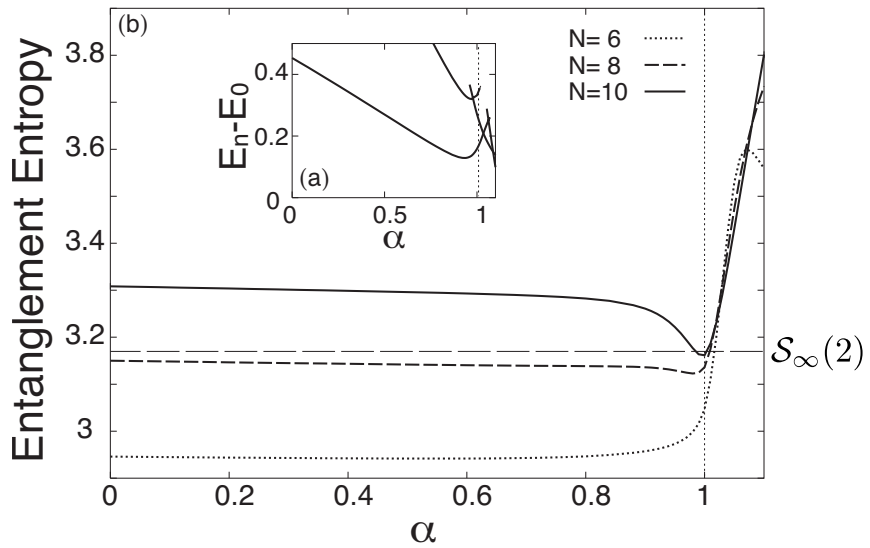

FIG. 2. (a) Energy gaps between the ground and the lowest two excited states in the system of $N=10$ sites with periodic boundary conditions. (b) The EE of the $S=2$ periodic $N=6,8,10$ Heisenberg model and its continuous deformations.

that this lower bound is closely related to the number of degrees of freedom emerging at the edge. In other words, if we can prepare a sufficiently long spin $S$ VBS chain with open boundaries, each edge state behaves as a free spin $S / 2$. This $(S+1)$-level system can be used as a qubit (qudit) for quantum computation by locally applying a magnetic field at the edge. The EE provides a typical measure for the quantum resources. We should note here that the EE has contributions not only from the edge state but also from the bulk except for the AKLT point. In this meaning, the AKLT point is a special point since the EE has a contribution only from the edges created by taking partial trace. This fact is related to the minimum correlation length at the AKLT point. It is also interesting that the EE at the $S=2$ AKLT point takes the minimum value. A similar behavior has been observed in the case of $S=1{ }^{24}$ Thus, we can conjecture that the EE takes a minimum value at the AKLT point in general SU(2)invariant models with integer spin $S$ as far as the edge-state picture is valid.

The authors are grateful to K. Azuma, H. Song, S. Murakami, S. Todo, and N. Nagaosa for fruitful discussions. The computation in this work has been done using the facilities of the Supercomputer Center, Institute for Solid State Physics, University of Tokyo. This work was supported by Grantin-Aid (Grant Nos. 15104006, 16076205, and 17105002) and NAREGI Nanoscience Project from the Ministry of Education, Culture, Sports, Science, and Technology. H.K. was supported by the Japan Society for the Promotion of Science. Y.H. was supported by Grant-in-Aid for Scientific Research, No. 17540347 from JSPS, No. 18043007 on Priority Areas from MEXT, and the Sumitomo Foundation.
*Electronic address: katsura@appi.t.u-tokyo.ac.jp

†Electronic address: hirano@pothos.t.u-tokyo.ac.jp

†Electronic address: hatsugai@ pothos.t.u-tokyo.ac.jp;

hatsugai@sakura.cc.tsukuba.ac.jp

${ }^{1}$ G. Vidal, J. I. Latorre, E. Rico, and A. Kitaev, Phys. Rev. Lett. 90, 227902 (2003).

${ }^{2}$ M. Levin and X. G. Wen, Phys. Rev. Lett. 96, 110405 (2006).

${ }^{3}$ A. Kitaev and J. Preskill, Phys. Rev. Lett. 96, 110404 (2006).

${ }^{4}$ S. Ryu and Y. Hatsugai, Phys. Rev. B 73, 245115 (2006).

${ }^{5}$ Y. Hatsugai, J. Phys. Soc. Jpn. 74, 1374 (2005); 75, 123601 (2006).

${ }^{6}$ F. D. M. Haldane, Phys. Lett. 93A, 464 (1983).

${ }^{7}$ F. D. M. Haldane, Phys. Rev. Lett. 50, 1153 (1983).

${ }^{8}$ I. Affleck, T. Kennedy, E. H. Lieb, and H. Tasaki, Phys. Rev. Lett. 59, 799 (1987).

${ }^{9}$ I. Affleck, T. Kennedy, E. Lieb, and H. Tasaki, Commun. Math. Phys. 115, 477 (1988).

${ }^{10}$ F. Verstraete and J. I. Cirac, Phys. Rev. A 70, 060302(R) (2004).

${ }^{11}$ W. D. Freitag and E. Müller-Hartmann, Z. Phys. B: Condens. Matter 83, 381 (1991).

${ }^{12}$ F. Verstraete, M. A. Martín-Delgado, and J. I. Cirac, Phys. Rev. Lett. 92, 087201 (2004).
${ }^{13}$ H. Fan, V. Korepin, and V. Roychowdhury, Phys. Rev. Lett. 93, 227203 (2004).

${ }^{14}$ J. P. Renard, M. Verdaguer, L. P. Regnault, W. A. C. Erkelens, J. Rossat-Mignod, and W. G. Stirling, Europhys. Lett. 3, 945 (1987).

${ }^{15}$ K. Katsumata, H. Hori, T. Takeuchi, M. Date, A. Yamagishi, and J. P. Renard, Phys. Rev. Lett. 63, 86 (1989).

${ }^{16}$ G. E. Granroth, M. W. Meisel, M. Chaparala, T. Jolicoeur, B. H. Ward, and D. R. Talham, Phys. Rev. Lett. 77, 1616 (1996).

${ }^{17}$ Y. Hatsugai, Phys. Rev. Lett. 71, 3697 (1993).

${ }^{18}$ A. Auerbach, Interacting Electrons and Quantum Magnetism (Springer, New York, 1998).

${ }^{19}$ D. P. Arovas, A. Auerbach, and F. D. M. Haldane, Phys. Rev. Lett. 60, 531 (1988).

${ }^{20}$ H. Fan, V. Korepin, and V. Roychowdhury, arXiv:quant-ph/ 0511150 (unpublished).

${ }^{21}$ M. Hagiwara, K. Katsumata, I. Affleck, B. I. Halperin, and J. P. Renard, Phys. Rev. Lett. 65, 3181 (1990).

${ }^{22}$ S. Miyashita and S. Yamamoto, Phys. Rev. B 48, 913 (1993).

${ }^{23}$ S. Qin, T. K. Ng, and Z. B. Su, Phys. Rev. B 52, 12844 (1995).

${ }^{24}$ T. Hirano and H. Hatsugai, arXiv:cond-mat/0703642, J. Phys. Soc. Jpn. (to be published). 